\title{
Análise da produção científica brasileira sobre nanotecnologia e saúde
} Analysis of brazilian scientific articles about nanotechnology and health

\section{Análisis de la producción científica brasileña acerca de nanotecnología y salud}

\author{
Maria Simone de Menezes Alencar | simone.alencar@unirio.br \\ Universidade Federal do Estado do Rio de Janeiro (Unirio), Programa de Pós Graduação em Biblioteconomia. \\ Rio de Janeiro, Brasil. \\ Rosany Bochner | rosany.bochner@icict.fiocruz.br \\ Fundação Oswaldo Cruz (Fiocruz), Instituto de Comunicação e Informação Científica e Tecnologia em Saúde (Icict). \\ Miriam Ferreira Freire Dias | miriamffdias@gmail.com \\ Universidade Federal do Estado do Rio de Janeiro (Unirio), Escola de Biblioteconomia. Rio de Janeiro, Brasil. \\ Adelaide Maria de Souza Antunes | $\underline{\text { adelaide@eq.ufrj.br }}$ \\ Universidade Federal do Rio de Janeiro (UFRJ), Centro de Tecnologia, Escola de Química. Rio de Janeiro, Brasil.
}

\section{Resumo}

No Brasil, desde o início dos anos 2000, há políticas públicas de incentivo à pesquisa dedicada à nanotecnologia. Este artigo apresenta estudo que visa investigar a produção científica brasileira em nanotecnologia e saúde, através da publicação de artigos científicos indexados na base de dados Web of Science, no período de 1995 a 2014. Através de análise bibliométrica e cientométrica, apoiada no uso do software VantagePoint, são identificados a evolução histórica da pesquisa no tema, as principais instituições brasileiras dedicadas ao objeto do estudo, a colaboração internacional e os temas de pesquisa pelos quais os autores brasileiros mais se interessam. Conclui-se que há um grande crescimento de publicações a partir do século XXI e uma diversificação de temas de pesquisa ao longo do período do estudo. As áreas de maior foco nas pesquisas mais recentes são farmacologia e farmácia, bioquímica, biologia molecular e cirurgia. Observa-se baixa internacionalização da pesquisa, com $30 \%$ dos artigos elaborados em colaboração com outros países.

Palavras-chave: nanotecnologia; bibliometria; cientometria; saúde; Brasil. 


\begin{abstract}
Since the early 2000s, Brazil has had public policies designed to incentive research in nanotechnology. This article presents a study designed to investigate Brazilian scientific literature about nanotechnology and health by means of the publication of scientific articles indexed in the Web of Science database from 1995 to 2014. Using bibliometric and scientometric analysis and a data mining software, VantagePoint, the historical evolution of research was identified, as well as the leading Brazilian institutions dedicating to studies such as, the international collaborations involving Brazilian researchers, and the research themes that have attracted their attention. The number of publications is growing more and more since the beginning of the 21th century and covering a diversification of research areas. The areas in which most research has been done recently are pharmacology \& pharmacy, biochemistry, molecular biology, and surgery. The internationalization of this area of research is relatively low: just $30 \%$ of the articles by Brazilian authors were coauthored by researchers from other countries.
\end{abstract}

Keywords: nanotechnology; bibliometrics; scientometrics; health; Brazil.

\title{
Resumen
}

En Brasil, desde principios de la década de 2000 existen políticas públicas para fomentar la investigación en nanotecnología. Este artículo presenta uno estudio que tiene como objetivo investigar la producción científica brasileña en la nanotecnología y la salud, a través de la publicación de artículos científicos indexados en la base de datos Web of Science, de 1995 a 2014. A través del análisis bibliométrica y cienciométrica, apoyada en el uso de software VantagePoint, se identifica evolución histórica de las investigaciones sobre el tema, las principales instituciones brasileñas dedicadas al estudio de la nanotecnología, la colaboración internacional y los temas de investigación que más despiertan el interés de los autores brasileños. De ello se desprende que hay un fuerte crecimiento de las publicaciones a partir del siglo XXI y la diversificación de los temas de investigación a lo largo del período de estudio. Las áreas de mayor enfoque en la investigación más reciente son la farmacología y farmacia, bioquímica y la biología molecular y la cirugía. Se observa una baja internacionalización de la investigación, con 30\% de los artículos escritos en colaboración con otros países.

Palabras clave: nanotecnología; bibliometría; cienciometria; salud; Brasil.

Contribuição dos autores:

Concepção e desenho do estudo: Maria Simone de Menezes Alencar e Rosany Bochner;

Aquisição, análise ou interpretação dos dados: Miriam Ferreira Freire Dias, Maria Simone de Menezes Alencar e Rosany Bochner; Redação do manuscrito: Maria Simone de Menezes Alencar e Rosany Bochner;

Revisão crítica do conteúdo intelectual: Adelaide Maria de Souza Antunes;

Declaração de conflito de interesses: não houve;

Fontes de financiamento: não houve;

Agradecimento/Contribuições adicionais: A realização da pesquisa aqui apresentada teve o apoio do CNPq, através de bolsa de pesquisador visitante da Fiocruz. Agradecemos também a Nanobusiness, que disponibilizou a licença de uso do software VantagePoint para a finalização da pesquisa.

Histórico do artigo: Submetido: 10.out.2016 | Aceito: 13.fev.2017 | Publicado: 31.mar.2017

Apresentação anterior: não houve;

Licença CC BY-NC atribuição não comercial. Com essa licença é permitido acessar, baixar (download), copiar, imprimir, compartilhar, reutilizar e distribuir os artigos, desde que para uso não comercial e com a citação da fonte, conferindo os devidos créditos de autoria e menção à Reciis. Nesses casos, nenhuma permissão é necessária por parte dos autores ou dos editores. 


\section{Introdução}

Os fundamentos do que hoje se denomina nanotecnologia foram inicialmente discutidos por Richard Feynman, conceituado físico norte-americano que, em 1959, afirmava que as leis que regem a dimensão atômica são fundamentalmente diferentes, permitindo novas possibilidades de funcionalidade das estruturas. O termo nanotecnologia, no entanto, foi cunhado em 1974 por Norio Taniguchi, professor da Tokyo Science University, referenciando-se a máquinas com níveis de tolerância menor que um mícron. O desenvolvimento de instrumentos capazes de gerar imagens reais de superfícies com resolução atômica no início da década de 1980 foram marcos importantes para a evolução dessa tecnologia. Desde então, estruturas e materiais nanotecnológicos têm sido desenvolvidos, promovendo inovações em diversos setores industriais. Para mais informações sobre a história da nanotecnologia, ver Bhat ${ }^{1}$; Alves$^{2}$; e Medeiros, Paterno, Mattoso ${ }^{3}$.

A nanotecnologia, segundo a International Standards Organization (ISO), contempla pelo menos um dos seguintes aspectos na sua caracterização: o (i) entendimento e controle da matéria e processos em nanoescala, tipicamente, mas não exclusivamente, abaixo de 100 nanômetros em uma ou mais dimensões, em que o aparecimento de fenômenos dependentes de tamanho permite novas aplicações; e a (ii) utilização das propriedades dos materiais em nanoescala que são diferentes das propriedades dos átomos individuais, moléculas, ou dos materiais macroscópicos, criando materiais, dispositivos e sistemas melhores que exploram essas novas propriedades ${ }^{4}$.

Dessa forma, pode-se considerar que a nanotecnologia é um campo científico multidisciplinar, que investiga novas propriedades que se manifestam em escala nanométrica para a manipulação e construção de novas estruturas. Abre oportunidades para a ciência, ao permitir a criação de materiais inéditos e inviáveis de serem produzidos sem essa tecnologia. Isso é possível porque, em escala atômica, as leis que regem a natureza são diferentes das que regem os objetos em escalas maiores.

Esse campo de estudo é considerado capaz de ajudar a solucionar diversos problemas nos setores industrial e econômico ${ }^{5}$. Na área da saúde, há perspectivas de avanços significativos no diagnóstico de doenças e produção de medicamentos mais eficazes e seguros ${ }^{6}$. Em nosso cotidiano, já utilizamos produtos com algum componente nanométrico, como chips, sensores, fibras de tecido, cosméticos, produtos esportivos e medicamentos.

Os estudos nanotecnológicos vêm atraindo investimentos em pesquisas em diversos países, provocando a expansão mundial do financiamento e da atividade na área nos últimos anos. Os Estados Unidos ea China lideram o ranking, seguidos da Alemanha, Japão e Coreia do Sul7. Em termos globais, mais de 60 países estabeleceram programas nacionais na área de nanotecnologia para aumentar a competitividade de suas empresas ${ }^{8}$.

No Brasil, os primeiros passos do financiamento para nanotecnologia ocorreram no início dos anos 2000, com a criação de redes e institutos de pesquisa financiados pelo Conselho Nacional de Desenvolvimento Científico e Tecnológico/Ministério da Ciência, Tecnologia e Inovação (CNPq/MCTI). A Política Industrial, Tecnológica e de Comércio Exterior (PITCE), lançada em 2004, marcou a retomada de políticas públicas voltadas para competitividade da indústria nacional, destacando a nanotecnologia como tecnologia portadora de futuro. Em 2005 foi lançado o Programa Nacional de Nanotecnologia, apoiado no Programa de Desenvolvimento da Nanociência e Nanotecnologia no âmbito do Plano Plurianual 2004-2007. Desde então, diversas políticas públicas federais envolvem esforços voltados para a nanotecnologia: Plano de ação em Ciência, Tecnologia e Inovação (CT\&I) (2007), Política de Desenvolvimento Produtivo (2008), Plano Brasil Maior (2011), Estratégia Nacional para CT\&I 2012-2015, culminando com a Iniciativa Brasileira de Nanotecnologia apresentada pelo MCTI em 2013. Essa iniciativa formalizou ações para criar, integrar e fortalecer as atividades governamentais, estimulando a interação com o setor privado para fomentar o desenvolvimento cientifico, tecnológico e inovativo na área de nanotecnologia.

Outra importante iniciativa nacional foi o desenvolvimento de estudo prospectivo, pela Agência Brasileira de Desenvolvimento Industrial (ABDI) e pelo Centro de Gestão e Estudos Estratégicos (CGEE), visando 
fomentar o desenvolvimento de políticas para nanotecnologia no país. Nesse estudo foram apresentados os setores que mais seriam impactados pela nanotecnologia. O setor de fabricação de material eletrônico e de aparelhos e equipamentos de comunicações estão no topo da lista; em seguida, estão os setores de medicina e saúde e de higiene, perfumaria e cosméticos9.

Delinear os limites de uma tecnologia emergente é fundamental para a compreensão dos caminhos de investigação e perspectivas de comercialização; entretanto, não é uma tarefa simples, sobretudo quando se trata de um campo complexo, transversal e com crescimento tão rápido como a nanotecnologia7.

\section{Bibliometria e cientometria como método}

Para a solução de problemas desse tipo, propõe-se uma abordagem bibliométrica e cientométrica. MaciasChapula ${ }^{10}$ caracteriza a bibliometria como a métrica que trabalha com documentos, artigos, autores, entre outras coisas. Já a cientometria, de acordo com o mesmo autor, considera as medidas relativas a disciplinas, áreas, campos. Esses dois conceitos são utilizados devido à sobreposição conceitual que apresentam, a bibliometria pela análise dos artigos e a cientometria pelo foco na área de nanotecnologia na saúde.

A bibliometria pode ser utilizada para avaliação quantitativa de publicações científicas e tecnológicas, permitindo o estudo de suas relações e interações. São aplicadas para o estabelecimento ou fortalecimento de indicadores, para que seja traçado um perfil do mundo técnico-científico, tanto em âmbito nacional como internacional ${ }^{11}$. Por meio desses estudos é possível situar a produção em Ciência e Tecnologia (C\&T) de um país em comparação à produção mundial, de uma instituição em relação ao seu país, ou ainda, a produção individual de cientistas pesquisadores em relação à sua comunidade ${ }^{10}$.

O uso da bibliometria e da cientometria para avaliar o desenvolvimento científico e tecnológico global da nanotecnologia tem sido utilizado por diversos autores ${ }^{12-17}$. No entanto, os estudos métricos dedicados a aplicações nanotecnológicas relacionadas à área da saúde são mais raros. Alguns estudos focados em uma subárea relacionada à saúde foram identificados, mas nenhum com o escopo das ciências da saúde como um todo. Por exemplo, Ostrowski, Martin, Conti, Hurt e Harthorn ${ }^{18}$ realizaram estudo bibliométrico dedicado à nanotoxicologia, área que busca entender a toxicidade de nanomateriais e produtos nanotecnológicos. Sua pesquisa envolveu artigos de periódicos indexados no SciFinder ${ }^{\mathrm{i}}$ e no Medline ${ }^{\mathrm{ii}}$ no período de 2000 a 2007, para elucidar a distribuição da pesquisa publicada em nanotoxicologia pelas diferentes áreas de saúde humana e meio ambiente, quais temas são emergentes e que materiais e rotas de exposição têm sido pesquisados. Mais recentemente, Wang, Yang, Yang, Long e $\mathrm{Li}^{19}$ analisaram o risco de nanomateriais engenheirados, através de análise das publicações indexadas na Web of Science no período de 1999-2012, identificando o crescente interesse nessa área de pesquisa.

Outro enfoque encontrado em estudos bibliométricos com nanotecnologia é a abordagem geográfica. Guan e $\mathrm{Ma}^{20}$ comparam o desenvolvimento da pesquisa em nanotecnologia na China com quatro outros países (França, Alemanha, Japão e Estados Unidos). Tang e Shapira ${ }^{8}$ analisam o padrão e a dinâmica de colaboração científica entre China e Estados Unidos. Alencar, Porter e Antunes ${ }^{21}$ analisam o patenteamento global da nanotecnologia a partir da avaliação do posicionamento de países entre os elos de uma cadeia de valor composta por nanomatérias-primas, nanointermediários e nanoprodutos.

A pesquisa aqui apresentada combina abordagens temática e geográfica, investigando o desenvolvimento da pesquisa produzida no Brasil em nanotecnologia com foco na saúde, através dos artigos científicos indexados na Web of Science nos últimos 20 anos (1995-2014).

i Disponível em: https://scifinder.cas.org.

ii Disponível em: https://www.ncbi.nlm.nih.gov/pubmed. 


\section{Recuperação da informação em nanotecnologia}

Arora, Porter, Youtie e Shapira7 discorrem sobre a necessidade da compreensão do domínio e evolução de tecnologias emergentes. Segundo os autores, pesquisadores têm utilizado várias estratégias de busca para identificar a literatura dedicada à nanotecnologia, incluindo desde a busca simples pelo prefixo nano, até abordagens mais complexas para a recuperação e análise dessas publicações.

Uma questão comum entre esses pesquisadores é a preocupação de encontrar o equilíbrio entre a revocação e a precisão, isto é, recuperar a maioria se não todos os registros sem ruídos (material não relevante) ou o silêncio (material pertinente não recuperado) ${ }^{7,13}$. Os conceitos de revocação e precisão costumam estar inversamente relacionados, ou seja, a recuperação de um grande volume de documentos está ligada à baixa precisão desses registros ${ }^{22}$.

Em busca de encontrar termos que recuperem o maior número de trabalhos pertinentes, foram desenvolvidas, por inúmeros pesquisadores, diferentes abordagens para elaboração de uma estratégia de busca que alcance a revocação e precisão desejadas. Huang, Notten e Rasters ${ }^{13}$ analisaram essas abordagens, que serão detalhadas a seguir, classificando-as em: consulta lexical, consulta lexical evolutiva, análise de citação e estratégia de análise de núcleo de revistas.

Uma consulta lexical baseia-se em pareceres de peritos para a seleção de palavras-chave. Embora considerada uma abordagem relativamente simples, os autores admitem que sua eficácia depende da proficiência dos peritos consultados.

Uma consulta lexical evolutiva emprega processos semiautomatizados de identificação de termos de alta ocorrência no resumo, título e campos de palavras-chave de artigos de periódicos correspondentes ao tema da pesquisa. Em seguida, os especialistas avaliam a lista dos termos encontrados e recomendam os mais adequados. Nas duas abordagens lexicais citadas, a subjetividade dos especialistas e a influência dos termos pré-selecionados podem comprometer a eficiência da estratégia ${ }^{13}$.

A estratégia de pesquisa baseada em análise de citações se apoia em um conjunto inicial de registros focados para identificar outros que citam e são citados por esse conjunto inicial de artigos. Através de algoritmos, cria-se uma sequência de ramos a partir das citações, com parâmetros que ajustam o equilíbrio entre a especificidade e a cobertura das publicações. O ponto fraco dessa abordagem está, segundo os autores, na dificuldade de reproduzir a estratégia devido ao próprio algoritmo do pesquisador e à necessidade de ter acesso a amplas bases de dados com altos custos de licenciamento. Essa abordagem foi também adotada por Zitte Bassecoulard ${ }^{23}$ e Mogoutove Kahane ${ }^{24}$, entre outros.

Por fim, as estratégias baseadas em um núcleo de periódicos analisam um pequeno número de revistas dedicadas à nanotecnologia e por meio de citação e análise de rede esse número é expandido. Teoricamente a precisão deve ser alta; entretanto, a revocação é baixa, uma vez que as publicações em nanotecnologia não se restringem apenas a revistas devotadas ao tema.

Conclui-se, portanto, que a estratégia proposta por Arora, Porter, Youtie e Shapira ${ }^{7}$, uma evolução de estratégia anterior desenvolvida por Porter, Youtie, Shapira e Schoeneck ${ }^{25}$, se apresenta como a mais completa. Essa versão é caracterizada como

uma estratégialexicalevolucionária,jáque emprega canais defeedbackentreoprocesso deidentificação de palavras-chave e a obtenção da opinião de especialistas. Nossa abordagem para modificar a lista de palavras-chave foi realizada através (1) avaliação sistemática, semiautomatizada de palavras-chave de alta ocorrência e (2) entrevistas, questionários e outras fontes de dados. (tradução nossa) 


\section{Metodologia}

A pesquisa, caracterizada como cientometria pelo uso da nanotecnologia na área da saúde e como estudo bibliométrico, foi realizada em três etapas, apresentadas a seguir: coleta, tratamento de dados e análise dos resultados.

\section{Coleta}

Foi realizado um estudo exploratório, que utilizou como fonte de informação a base de dados Web of Science iii, disponível no Portal de Periódicos da Capes ${ }^{\text {iv }}$. Além de ser multidisciplinar, ter cobertura temporal ampla e seletividade, essa fonte possui uma característica fundamental para os objetivos deste estudo: a completude de endereço, ou seja, os endereços de todos os autores são registrados, permitindo a identificação da instituição e do país de origem de todos os colaboradores do artigo.

A estratégia de busca a ser adotada exigia a combinação de dois conceitos amplos: nanotecnologia e saúde. Para a área de nanotecnologia, o estudo adotou a estratégia modular, ou seja, módulos para inclusão de termos e conceitos de busca e para exclusão de registros indesejados, proposta por Porter, Youtie, Shapira e Schoeneck ${ }^{25}$ e apresentada na Figura 1.

\begin{tabular}{|c|c|c|c|}
\hline \multicolumn{4}{|c|}{ STEP 1: INCLUSION TERMS AND MODULAR QUERY COMPONENTS } \\
\hline \multirow{7}{*}{$\begin{array}{l}\text { A - Search title, abstract } \\
\text { and keyword fields }\end{array}$} & A1 & \multicolumn{2}{|l|}{ Nano* } \\
\hline & A2 & \multicolumn{2}{|c|}{ Quantum (i.e.terms including "quantum dot") } \\
\hline & A3 & \multicolumn{2}{|c|}{ Self-assembly (contingent on a broad array of other terms) } \\
\hline & A4 & \multicolumn{2}{|c|}{ Other nanotechnology terms not captured by nano } \\
\hline & A5 & \multicolumn{2}{|c|}{ Microscopy terms (contingent on a broad array of other terms) } \\
\hline & A6 & \multicolumn{2}{|c|}{ Nano-pertinent (contingent on a broad array of other terms) } \\
\hline & A7 & \multicolumn{2}{|c|}{ Nano-pertinent (contingent on a narrow array of other terms) } \\
\hline B - Search specific journals & B1 & \multicolumn{2}{|c|}{ Artcles belonging to specific nanotechnology journals } \\
\hline \multicolumn{4}{|l|}{ STEP 2 - EXCLUSION TERMS } \\
\hline \multicolumn{3}{|c|}{ A - articles with specific non-nano terms } & \multirow{2}{*}{ ARE REMOVED } \\
\hline \multicolumn{3}{|c|}{$\mathrm{B}$ - articles with only nano* measurement terms } & \\
\hline
\end{tabular}

Figura 1 - Estratégia de busca adotada

Fonte: Porter, Youtie, Shapira e Shoeneck (2008) e Arora, Porter, Youtie e Shapira (2013).

Em relação ao segundo conceito envolvido na busca, a saúde, foi representado através da área de pesquisa 'ciências da vida e biomedicina'v

A Web of Science é composta por diversas tipologias documentais além de artigos científicos, tais como editoriais, artigos de revisão, cartas publicadas em periódicos indexados, comunicações apresentadas em

\footnotetext{
iii Disponível em: https://www.webofknowledge.com.

iv Disponível em: http://www.periodicos.capes.gov.br.

v O campo 'área de pesquisa', presente na Web of Science, apresenta uma categorização dos assuntos da base em cinco grandes áreas: Artes e Humanidades, Ciências da vida e Biomedicina, Ciências físicas, Ciências sociais e Tecnologia. As Ciências da vida e Biomedicina englobam 75 subáreas.
} 
eventos, entre outras publicações. Para o estudo aqui apresentado foram selecionados apenas os artigos de periódicos, pois, segundo Ziman ${ }^{26}$, esses são a principal evidência da produção de conhecimento devido à certificação gerada tanto pela revisão por pares como pelos editores dessas publicações.

Os resultados encontrados representama produçãocientíficaindexadanaWeb ofScienceparananotecnologia e saúde. Através de recursos básicos de análise da própria base foram feitas algumas considerações iniciais sobre a evolução temporal dessa produção e os países dos autores que publicam sobre a temática.

Em seguida foi feito o refinamento para que pelo menos um autor fosse de uma instituição localizada no Brasil. Essa fase foi realizada através da seleção de país de afiliação igual ao Brasil. Observou-se que a produção científica nacional se inicia em 1982, mas que até 1995 foram publicados apenas vinte artigos. Assim, optou-se pelo corte do período de 1995 a 2014, perfazendo 20 anos de publicações de artigos brasileiros ${ }^{\mathrm{vi}}$ em nanotecnologia e saúde para as análises subsequentes.

\section{Tratamento de dados}

Os dados bibliográficos dos resultados encontrados foram exportados, em formato texto, para importação

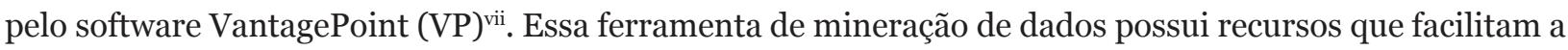
padronização de campos, a exclusão de itens repetidos e diversos recursos para geração de listas, matrizes e mapas de correlação de modo a facilitar a análise dos resultados.

No VantagePoint foi feita a verificação se havia itens duplicados ${ }^{\text {viii }}$ e a padronização dos campos de análise: instituição do autor, nome dos autores e países em que se localizam. Essa etapa é de fundamental importância, conforme apontado por Bochner, Alencar, Veiga e Machado ${ }^{27}$. O software possui instrumental que facilita essa padronização, exigindo, no entanto, extrema atenção na validação dos metadados.

Como o trabalho pretende analisar a internacionalização da pesquisa em nanotecnologia e saúde do Brasil, houve o cuidado metodológico de que todos os registros tivessem preenchido o campo país do autor. Os dados brutos, recuperados da Web of Science continham 99\% dos artigos com o país do autor. Para completar esse metadado foi utilizado o campo de ReprintAdress, sendo este unificado com o campo país do autor. Dessa forma, é possível analisar a completude dos registros em relação ao país dos autores.

Após a padronização do nome das instituições de autoria através de algoritmo do VP e análise manual, foi feito um corte naquelas que tinham pelo menos cinco artigos. Este conjunto, que representa $96 \%$ da amostra, foi classificado pela natureza da instituição: estrangeira e nacional. As nacionais foram classificadas em universidades federais e institutos federais de educação, universidades estaduais, universidades privadas e institutos de pesquisa e empresas.

Foram realizadas análises temporais de países com publicações em coautoria com o Brasil e colaboração internacional. Também são apresentadas as instituições nacionais envolvidas com o assunto, e as áreas de pesquisa com maior foco nas publicações nacionais.

Inicialmente, apresentamos um panorama da produção científica global em nanotecnologia e saúde, baseado nos resultados globais obtidos pela Web of Science. Em seguida, analisamos de forma mais focada a produção científica brasileira referente a essa temática.

vi Foi considerado como artigo brasileiro aquele que tem pelo menos um autor vinculado à instituição localizada no Brasil.

vii https://www.thevantagepoint.com/

viii Embora teoricamente não existam itens duplicados em uma mesma base de dados, estudos anteriores de Bochner, Alencar, Veiga e Machado (2012)27 apontaram essa existência devido a uma série de problemas na indexação dos documentos: grafia de nomes diferentes, artigo no prelo e artigo já publicado etc. 


\section{Resultados e discussão}

Esta seção apresenta os resultados da pesquisa e, concomitantemente, discute os mesmos, inicialmente no nível global e, em seguida, com o foco no Brasil.

Para obtenção dos resultados foi utilizada como estratégia de busca a consulta lexical, baseada em Arora, Porter, Youtie e Shapira ${ }^{7}$ e Porter, Youtie, Shapira e Schoeneck ${ }^{25}$. As buscas foram realizadas na Web of Science, identificando 1.342.435 registros sobre nanotecnologia, dos quais quase 83\% (1.107.804) referemse a artigos de periódicos. Esse resultado foi refinado com as áreas de pesquisa relacionadas às ciências da vida e biomedicina, gerando um conjunto de 104.952 artigos de periódicos, considerando autores de todas as nacionalidades. Os artigos de nanotecnologia e saúde, portanto, representam cerca de $9 \%$ do total de artigos publicados sobre nanotecnologia indexados na base.

Para uma visão global da pesquisa em nanotecnologia e saúde foi construída a Figura 2, que apresenta a evolução temporal da pesquisa voltada para o tema por década. Embora as pesquisas na área tenham se iniciado na década de 1990, o expressivo crescimento a partir do ano 2000 pode ser explicado pelas iniciativas nacionais de pesquisa em nanotecnologia que se tiveram início nessa década. Os Estados Unidos, precursores dessas iniciativas, lançaram em 2000 a National Nanotechnology Initiative (NNI), sendo seguidos nos anos seguintes pelos demais países em desenvolvimento. Cabe destacar também que a produção científica dos últimos cinco anos já ultrapassa toda a publicação na década anterior.

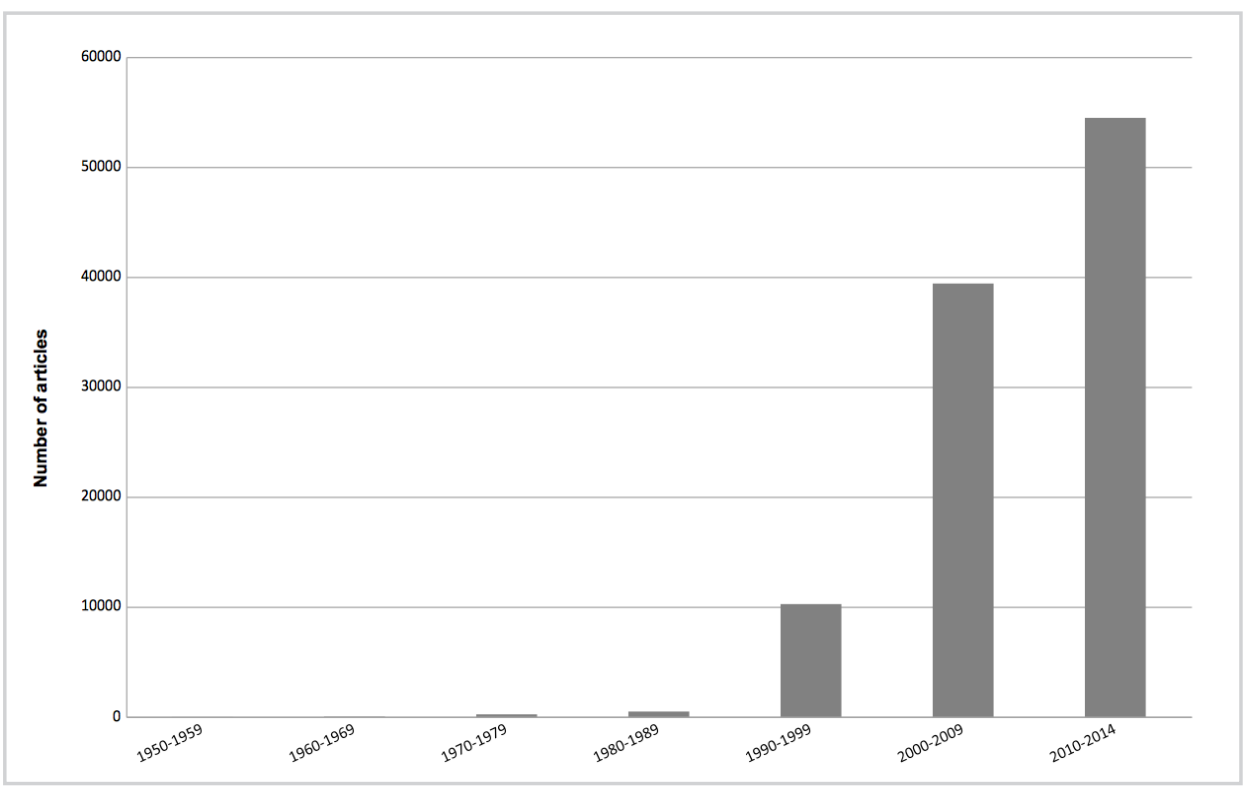

Figura 2 - Evolução histórica da publicação voltada para a nanotecnologia e saúde, por década Fonte: Elaborada pelas autoras (2017).

A análise de autoria aponta como principais países os Estados Unidos, seguidos pela China, Alemanha e Japão. Este resultado também foi encontrado por Arora, Porter, Youtie e Shapira ${ }^{7}$, em relação à nanotecnologia como um todo, mostrando que na área de saúde esses países também são líderes.

Com relação ao país dos autores, observa-se que o Brasil se encontra em $17^{\circ}$ lugar no ranking do qual os 20 primeiros são apresentados na Figura 3. 


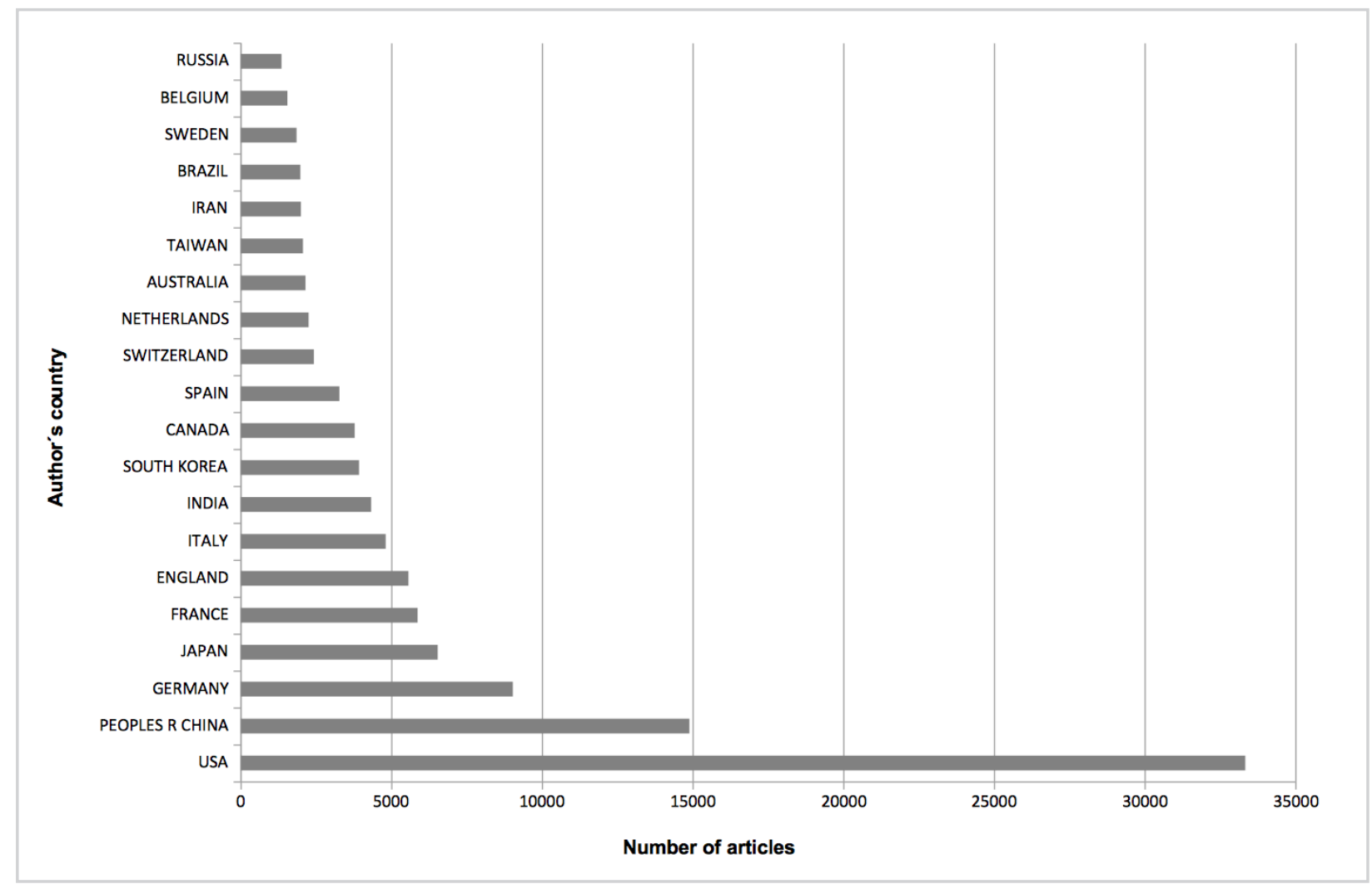

Figura 3 - Principais países com publicações em Nano \& Saúde Fonte: Elaborada pelas autoras (2017).

\section{Análise da produção brasileira voltada para nanotecnologia e saúde}

A seguir apresentam-se os resultados referentes à produção científica do Brasil voltada para nanotecnologia e saúde, considerada aqui como os artigos publicados por pelo menos um autor vinculado a uma instituição localizada no país.

\section{Evolução histórica}

Foi gerado um subconjunto de 1.980 artigos de periódicos, com pelo menos um autor vinculado a alguma instituição localizada no Brasil. Após a eliminação de duplicatas ${ }^{\mathrm{ix}}$, o universo de análise continha 1.956 artigos sobre nanotecnologia e saúde publicados por autores brasileiros no período de 20 anos (19952014). A Figura 4 apresenta a evolução histórica dessas publicações no país.

O eixo à esquerda, representado no gráfico pelas colunas, apresenta o número absoluto de artigos por ano. A aparente redução no ano de 2014 se deve ao fato de a coleta de dados ter sido realizada em dezembro do ano citado, não contendo, portanto, toda a produção daquele ano. O eixo à direita, representado pela linha, apresenta o número acumulado de artigos, que indica um crescimento exponencial de publicações, com tendência a uma maior inclinação a partir de 2007.

ix A duplicidade ocorre quando um mesmo artigo se apresenta em dois registros distintos. Em geral, isso acontece quando ele é indexado no prelo e, posteriormente, com os dados finais da edição. 


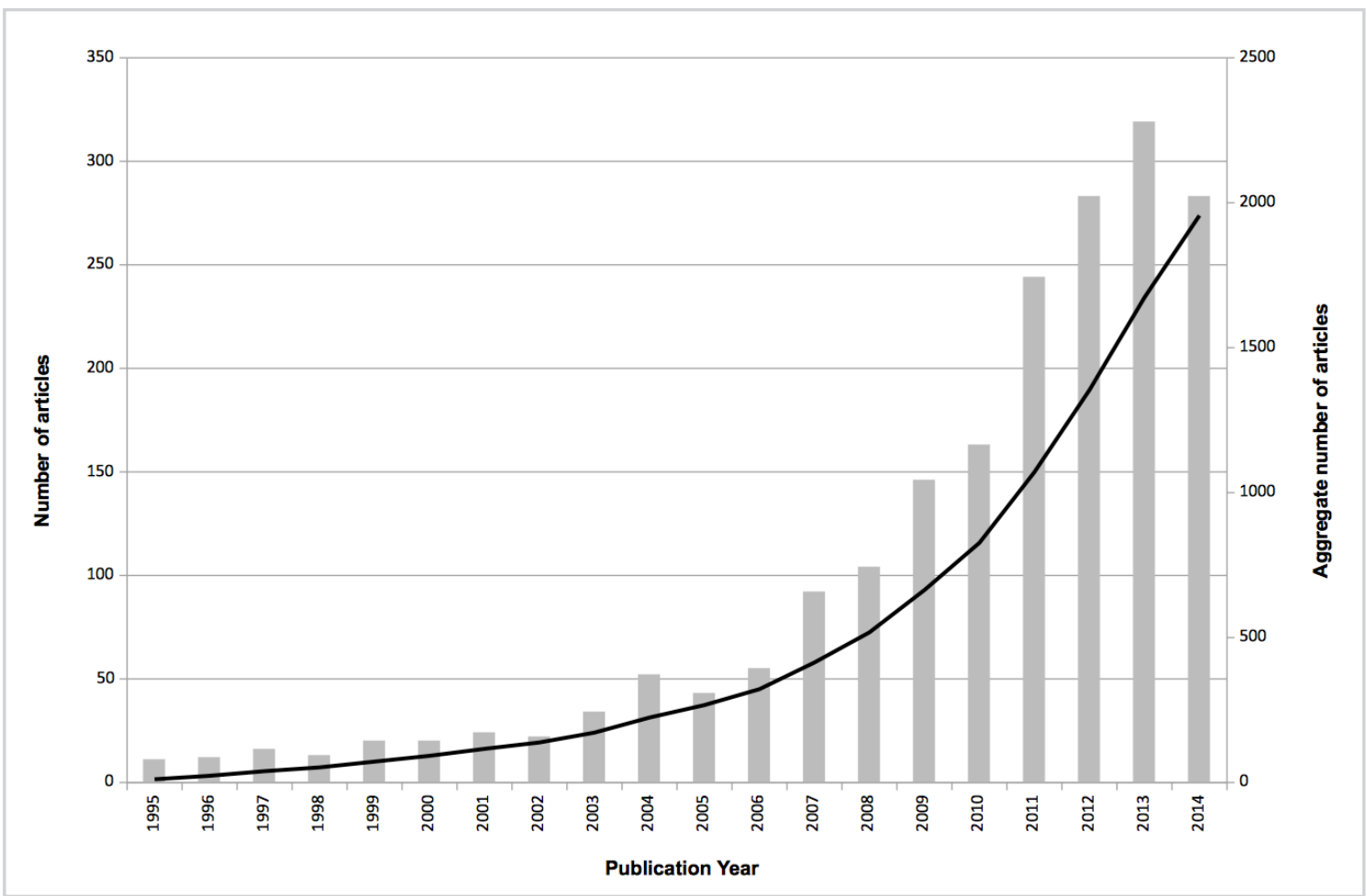

Figura 4 - Evolução histórica da publicação brasileira sobre nanotecnologia e saúde Fonte: Elaborada pelas autoras (2017).

\section{Países com instituições coautoras às do Brasil}

Em cerca de 70\% dos artigos não há coautoria internacional; apenas 592 artigos (30\%) foram redigidos em parceria com autores de outros países. Este resultado permite afirmar que há baixa internacionalização da pesquisa voltada para nanotecnologia e saúde no Brasil.

A figura 5 apresenta a distribuição geográfica dos países com publicações sobre nanotecnologia e saúde, no período de 1995 a 2014, redigidas em parceria com autores brasileiros. A cor mais intensa indica maior número de artigos. Observa-se que o país com mais coautoria é os Estados Unidos, seguido da França, Alemanha e Portugal.

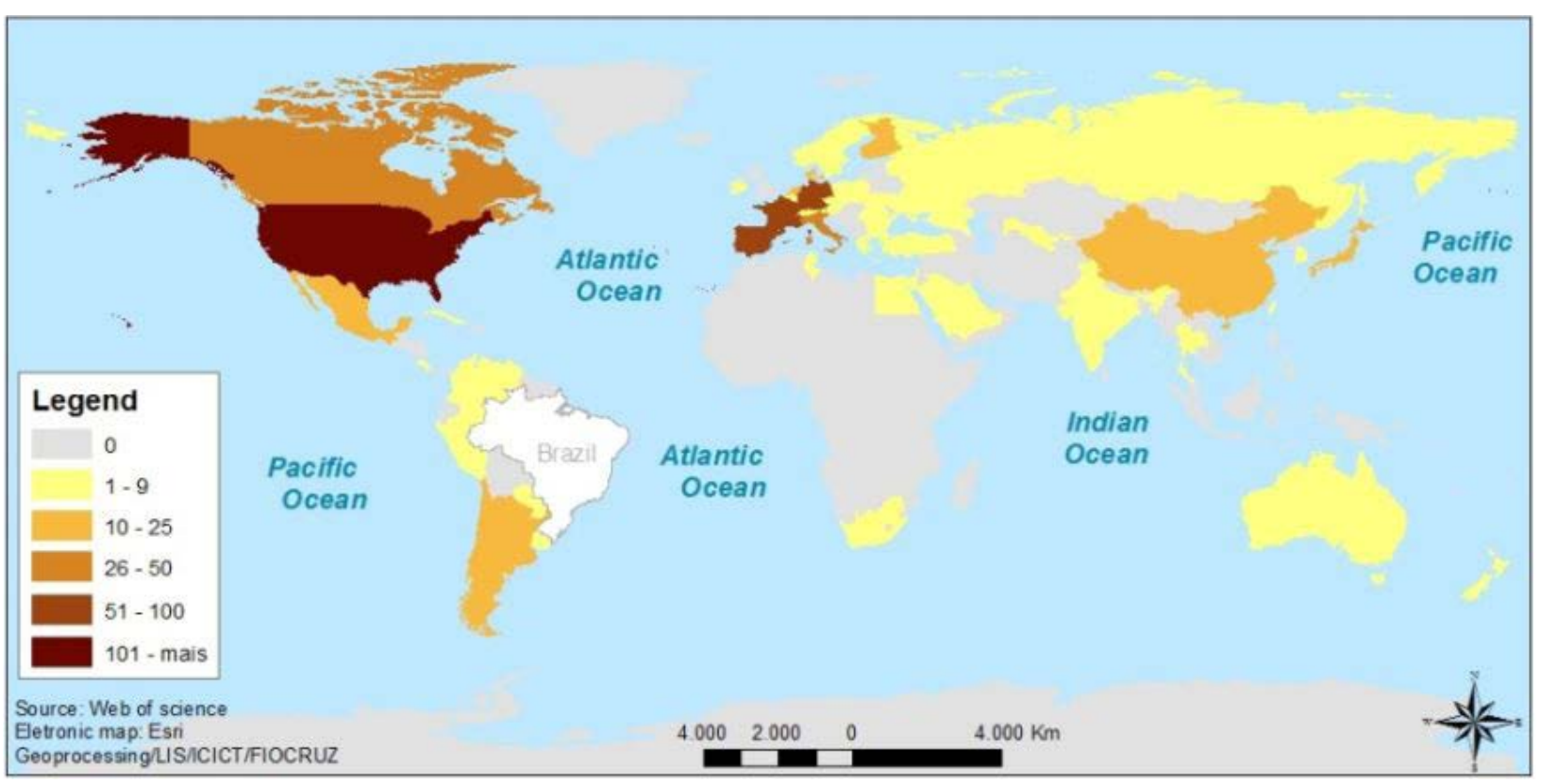

Figura 5 - Países com publicações em coautoria com o Brasil Fonte: Elaborada pelas autoras (2017). 


\section{Principais instituições}

Foram selecionadas as instituições com cinco ou mais artigos, que representam 96\% da amostra. Esse conjunto contém 117 instituições. Destas, 35 são estrangeiras e 82 são nacionais.

Entre as instituições nacionais, encontram-se 38 universidades federais, 17 universidades particulares, 14 universidades estaduais, nove institutos de pesquisa e duas empresas. Embora as universidades federais sejam em maior número, e apresentem o maior número de artigos (1.148), a média de artigos é de 30 ao passo que as universidades estaduais, que produziram no mesmo período 930 artigos, com média de 66 documentos.

As universidades estaduais - Universidade de São Paulo (USP), Universidade Estadual de Campinas (Unicamp), Universidade Estadual Paulista (Unesp) - que se destacam concentram-se no estado de São Paulo, refletindo o investimento que aquele estado faz em pesquisa.

O Quadro 1 apresenta as vinte principais instituições que publicam em nanotecnologia e saúde, no período de 1995 a 2014, e sua respectiva tipologia.

Quadro 1 - Principais instituições dos autores e sua tipologia

\begin{tabular}{|c|c|c|}
\hline No de artigos & Afiliação do autor & Tipologia \\
\hline 489 & Univ de Sao Paulo - USP & universidade estadual \\
\hline 252 & Univ Estadual de Campinas - Unicamp & universidade estadual \\
\hline 198 & Univ Fed Rio Grande Sul - UFRGS & universidade federal \\
\hline 191 & Univ Estadual Paulista - Unesp & universidade estadual \\
\hline 154 & Univ Fed do Rio de Janeiro - UFRJ & universidade federal \\
\hline 114 & Univ Fed de Minas Gerais - UFMG & universidade federal \\
\hline 107 & Univ Fed de Santa Catarina - UFSC & universidade federal \\
\hline 73 & Univ Fed de São Carlos - UFSCar & universidade federal \\
\hline 70 & Univ Fed de São Paulo - Unifesp & universidade federal \\
\hline 66 & Univ Fed do Ceará - UFC & universidade federal \\
\hline 66 & Univ Fed de Pernambuco - UFPE & universidade federal \\
\hline 65 & Empresa Bras. de Pesq. Agropecuária - Embrapa & inst. de pesquisa federal \\
\hline 53 & Univ de Brasília - UnB & universidade federal \\
\hline 48 & Fundacãoo Oswaldo Cruz - Fiocruz & inst. de pesquisa federal \\
\hline 42 & Univ Fed de Santa Maria - UFSM & universidade federal \\
\hline 36 & Univ Fed Fluminense - UFF & universidade federal \\
\hline 34 & Univ Fed do Paraná- UFPR & universidade federal \\
\hline 32 & Univ Estadual de Ponta Grossa - UEPG & universidade estadual \\
\hline 32 & Univ Fed de Uberlândia - UFU & universidade federal \\
\hline 31 & Univ Fed do Rio Grande do Norte - UFRN & universidade federal \\
\hline
\end{tabular}

Fonte: Elaborado pelas autoras (2017).

\section{Evolução da colaboração internacional}

Para avaliar a evolução da colaboração internacional ao longo do tempo do estudo, os artigos foram classificados em produzidos somente por brasileiros e aqueles com coautoria internacional. 
A Figura 6 mostra as principais instituições autoras e a relação entre a produção com coautores estrangeiros e brasileiros. Destacam-se a Fiocruz e a Universidade Federal do Ceará, ambas com cerca de $40 \%$ de sua produção com parcerias internacionais.

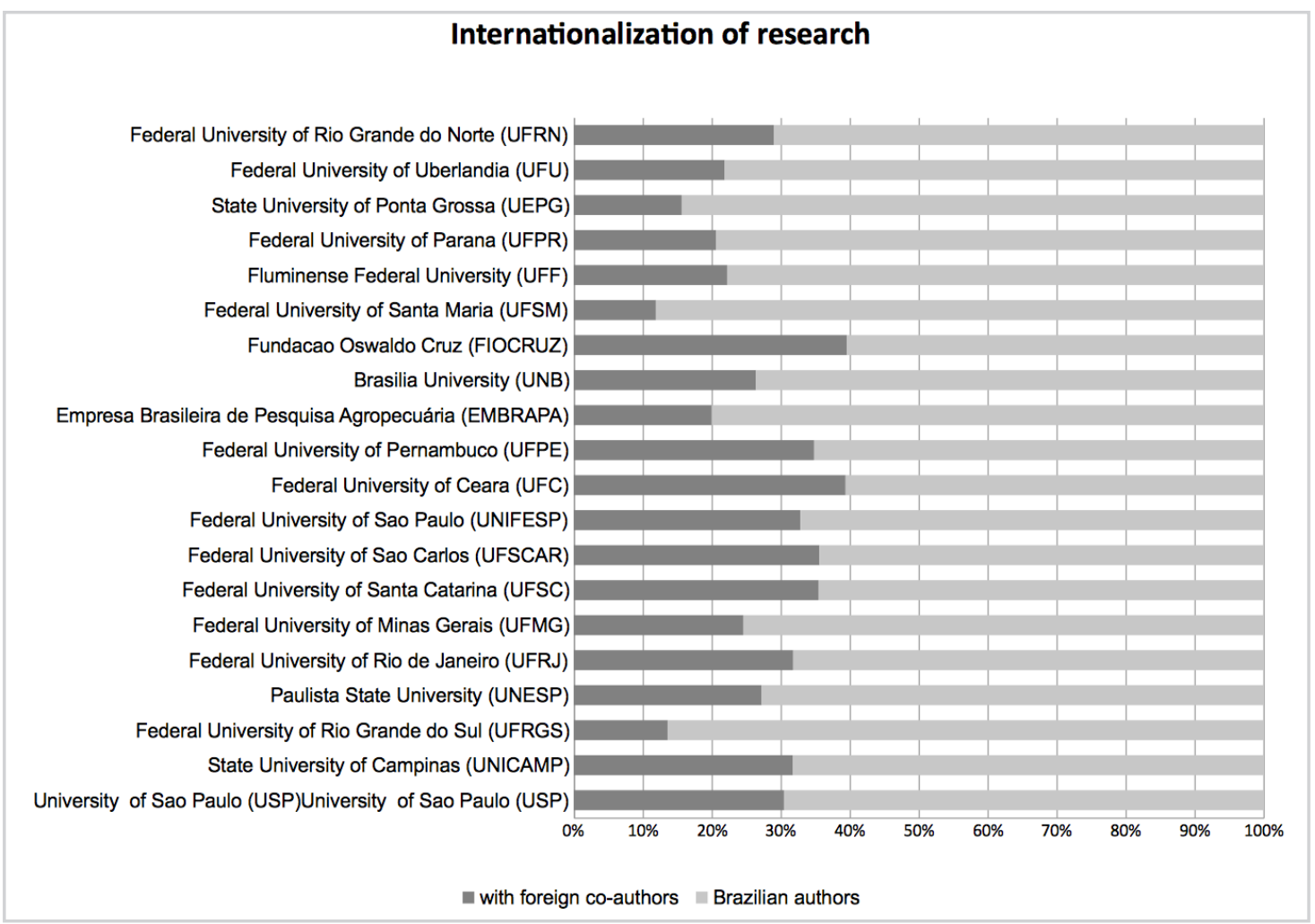

Figura 6 - Distribuição de coautoria brasileira e estrangeira nas principais instituições autoras Fonte: Elaborada pelas autoras (2017).

\section{Temas de pesquisa}

A observação das 75 áreas de pesquisa relacionadas às ciências da vida e biomedicina mostra que 65 temas são contemplados ${ }^{\mathrm{x}}$. A análise dos temas ao longo do tempo mostra forte diversificação da pesquisa, conforme é possível ver na Figura 7.

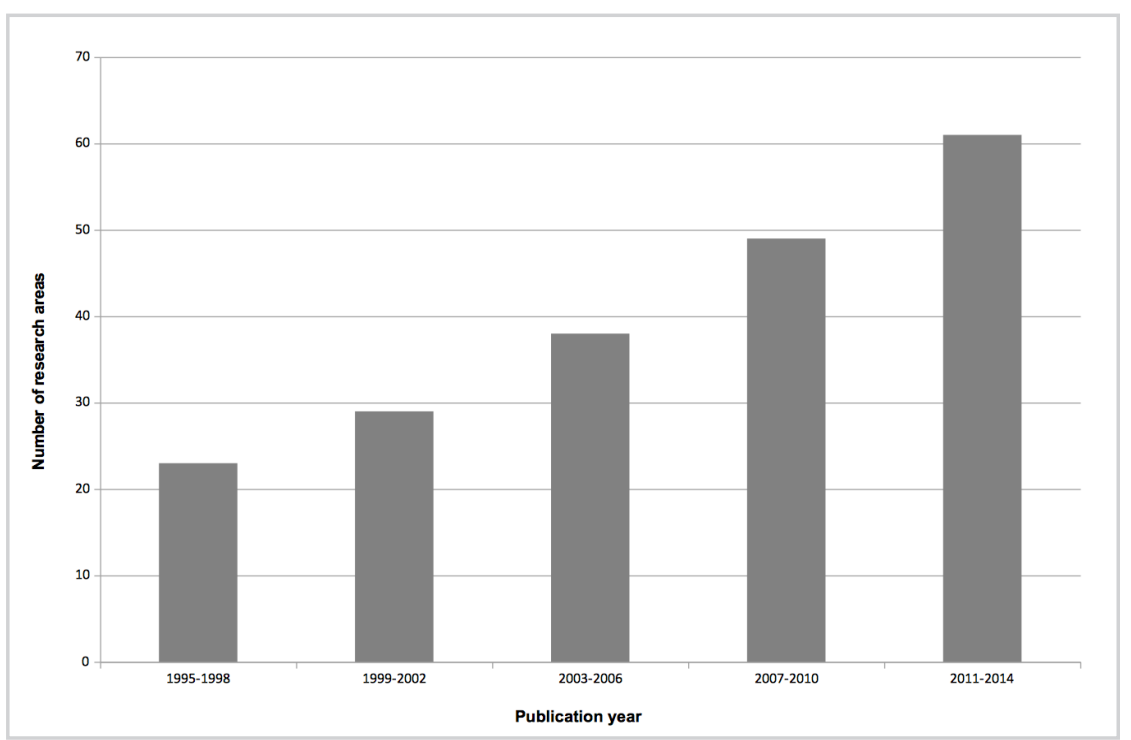

Figura 7 - Evolução do número de áreas de pesquisa por quadriênio

x As 10 áreas de pesquisa relacionadas às ciências da vida e medicina que não tiveram artigos publicados no período do estudo são: alergia, medicina intensiva, medicina de urgência, medicina legal, outros tópicos de ciências biomédicas da vida, ética médica, enfermagem, reabilitação, abuso de substâncias químicas, biodiversidade e conservação. 
Fonte: Elaborada pelas autoras (2017).

Destacam-se na Figura 8 a evolução temporal dos 22 temas com mais de 20 artigos, que representam 93\% do total de artigos analisados.

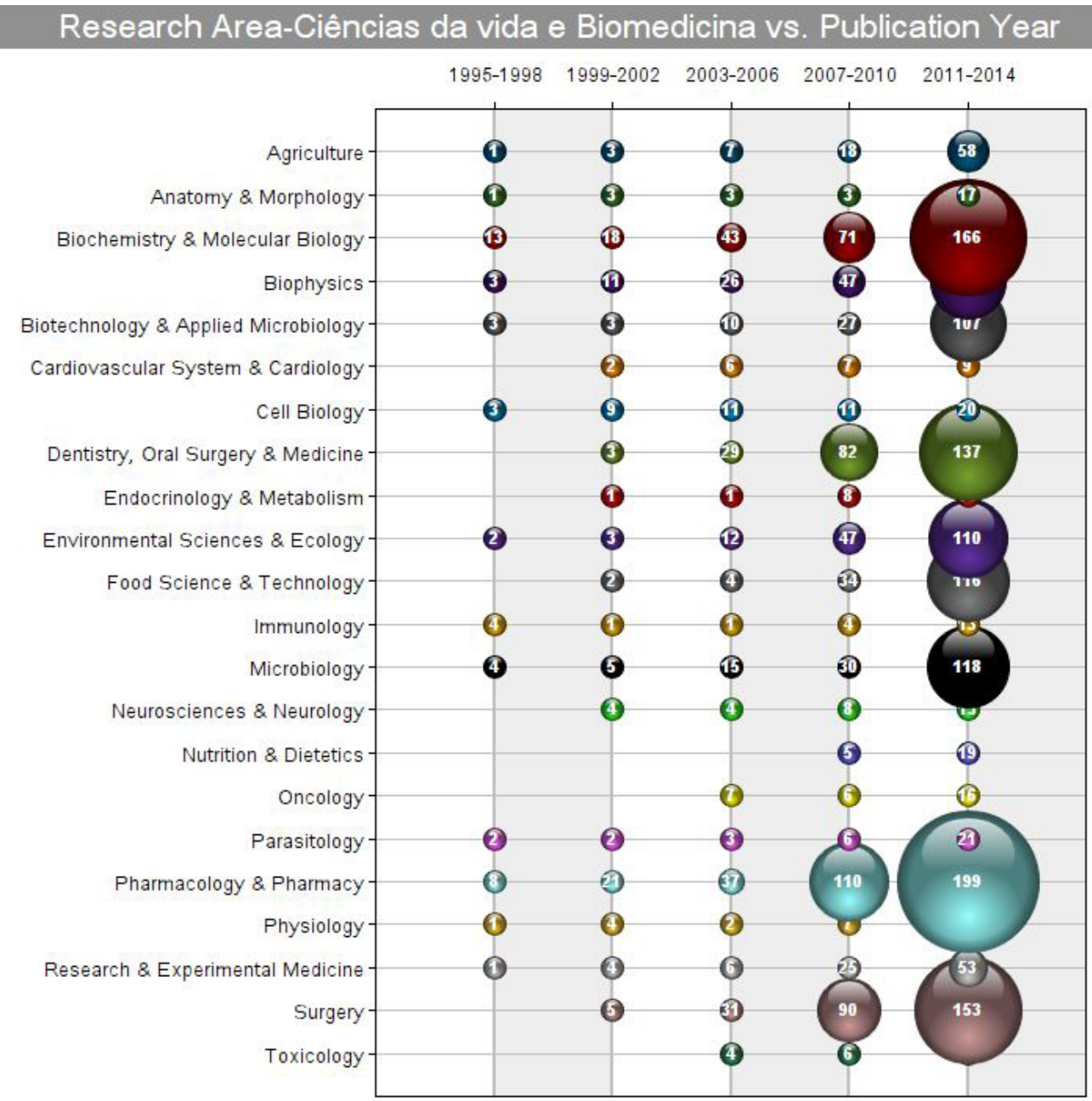

Figura 8 - Evolução dos temas de pesquisa por quadriênio

Fonte: Elaborada pelas autoras (2017).

É possível observar áreas com grande crescimento, como farmacologia e farmácia, bioquímica e biologia molecular e cirurgia.

\section{Análise dos temas de pesquisa por origem de autoria}

Como na análise global, foi possível observar que 70\% dos artigos são publicados só por brasileiros, optou-se por verificar como se comporta essa proporção nas principais áreas de pesquisa.

Considerando artigos de autoria só de brasileiros versus autoria com estrangeiros, a Figura 9 apresenta a distribuição percentual por tema. Observa-se que há áreas - como Dentistry, Oral SurgeryeMedicine; NeuroscienceseNeurology e Surgery - em que a colaboração com autores de outros países é maior do que a média de 70\%.

É importante destacar que um mesmo artigo pode estar classificado em mais de uma área de pesquisa, visto que esta é indexada através do periódico. 


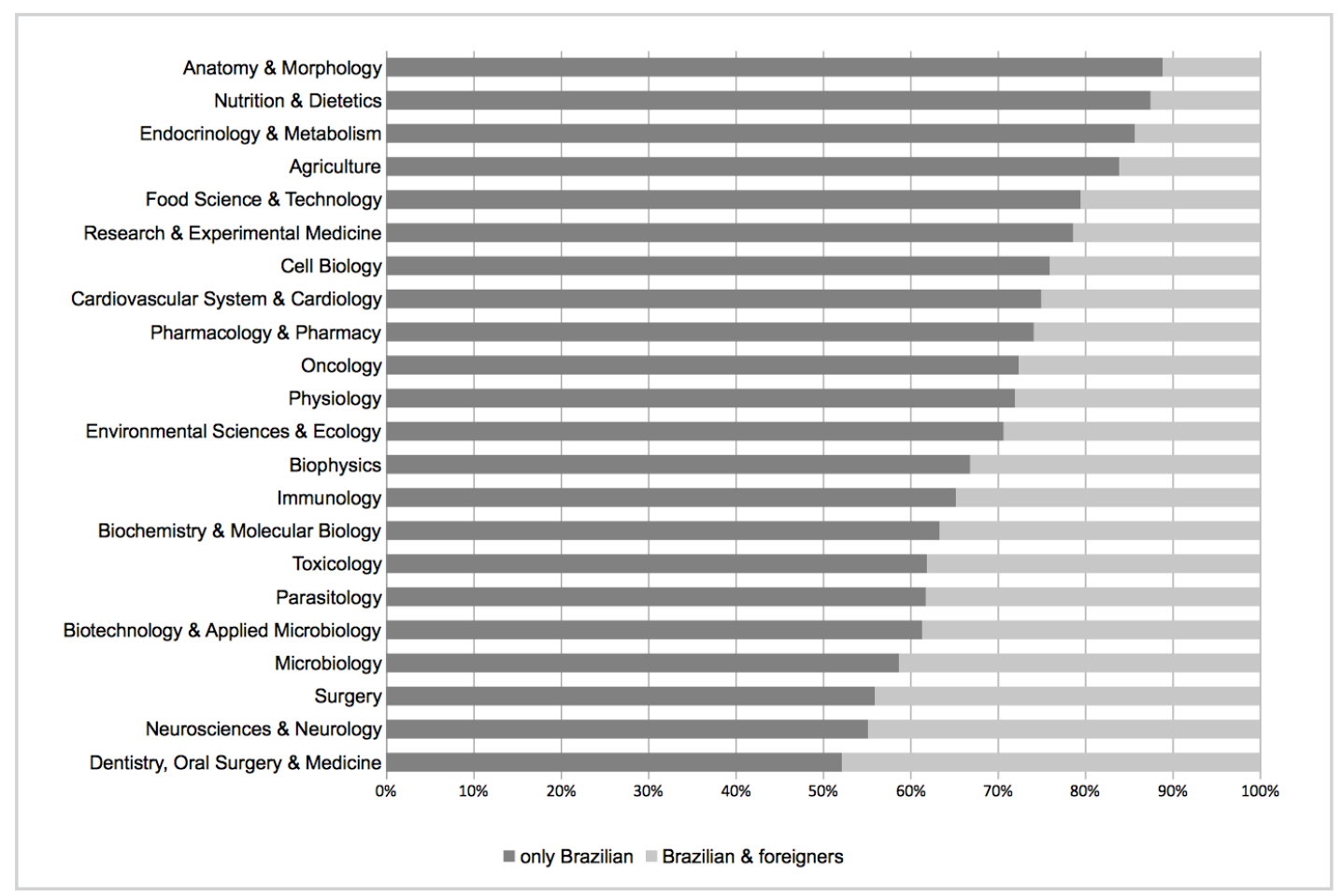

Figura 9 - Distribuição da origem dos autores por tema de pesquisa Fonte: Elaborada pelas autoras (2017).

\section{Conclusões}

A partir deste novo milênio, o mundo e o Brasil vêm considerando a nanotecnologia como área estratégica. No Brasil essa consideração é facilmente demonstrada através dos programas de governo, entre outros: a Política Industrial, Tecnológica e de Comércio Exterior (PITCE), a criação de redes e institutos de pesquisa do CNPq, a Política de Desenvolvimento Produtivo (PDP), e o Plano Brasil Maior (PBM). A nanotecnologia é transversal e impacta vários setores incluindo a saúde, e é possível prever que essa tecnologia pode trazer avanços significativos na produção de medicamentos como, por exemplo, em liberação controlada no alvo desejado assim como no diagnóstico (kits e marcadores). Este trabalho permitiu verificar, através de análise bibliométrica das publicações, a produção no mundo e no Brasil sobre o uso da nanotecnologia na área da saúde.

Nesse sentido, o estado da arte do cenário brasileiro mostra que as universidades públicas são as que mais publicam na área, destacando-se instituições no estado de São Paulo, como a USP e a Unicamp, líderes em publicações, evidenciando a atuação forte do estado nesse setor. No entanto, observa-se que, do ponto de vista de parcerias internacionais em publicações, se destacam a Fundação Oswaldo Cruz e a Universidade Federal do Ceará.

Considerando os temas de pesquisa em que a nanotecnologia se insere na área da saúde, verifica-se uma diversificação presente em 65 temas dos 75 categorizados pela Web of Science como área da saúde. Algumas dessas áreas com forte crescimento do quadriênio 2007-2010 para o quadriênio 2011-2014: Biochemistry e Molecular Biology; Pharmacology e Pharmacy; Dentistry, Oral Surgery e Medicine; e Surgery. No último quadriênio (2007-2010) destacam-se Biophysics, Biotechnology e Applied Microbiology; Environmental Sciences e Ecology; Food Science e Technology; e Microbiology.

Apesar do crescimento desses temas de pesquisa, é possível constatar que 70\% das publicações não foram redigidas em coautoria com pesquisadores de outros países, o que indica ainda pouca parceria internacional. No entanto, observa-se que dentre as áreas crescentes duas se destacam em parcerias internacionais: Dentistry, Oral Surgery e Medicine; e Surgery. Além disso, chama a atenção que apesar de haver poucas publicações na área de Neurosciences e Neurology, mais da metade delas resulta de parcerias internacionais. 
Há 65 áreas da saúde em que 82 instituições brasileiras publicaram mais de cinco artigos nos últimos 20 anos. No entanto, é possível observar que somente nove áreas contemplam 1.212 artigos, que são objeto de produção significativa. Como continuidade deste estudo pretende-se analisar nessas nove áreas, as instituições brasileiras que têm potencial para parcerias do uso da nanotecnologia na área da saúde. Além disso, fazer o mesmo tipo de tratamento em relação ao patenteamento da nanotecnologia nessas áreas, visando identificar sinergias e gargalos entre a produção científica e tecnológica no país.

\section{Referências}

1. Bhat JSA. Heralding a new future: nanotechnology? CurrSci. 2003;85(2):147-54.

2. Alves OL. Nanotecnologia, nanociência e nanomateriais: quando a distância entre presente e futuro não é apenas questão de tempo. ParcEstrat. 2010;9(18):23-40.

3. Medeiros ES, Paterno LG, Mattoso LHC. Nanotecnologia. In: Duran N, Mattoso LHC, MoraisPC, editores. Nanotecnologia: introdução, preparação, e caracterização de nanomateriais e exemplos de aplicação. São Paulo: Artliber, 2006.

4. InternationalOrganization for Standardization. TC229:nanotechnologies. Geneva; 2012 [acesso em 2016jan.22]. Disponível em: http://www. iso.org/iso/iso technical committee.html?commid=381983.

5. lencar MSM, Bochner R, Dias MFF. Nanotecnologia em Ciências da Saúde no Brasil: um olhar informétrico sobre os grupos de pesquisa. Liinc R. 2013;9(1):47-65.

6. Rossi-Bergmann BA. Nanotecnologia: da saúde para além do determinismo tecnológico. Ci eCul. 2008;60(2):54-7.

7. Arora SK, Porter AL, Youtie J, Shapira P. Capturing new developments in na emerging technology: an updated search strategy for identifying nanotechnology research outputs. Scientometrics. 2013;95(1):351-70.

8. Tang L, Shapira P. China-US scientific collaboration in nanotechnology: patterns and dynamics. Scientometrics. 2011;88(1):1-16.

9. Agência Brasileira de Desenvolvimento Industrial. Estudo prospectivo de nanotecnologia. Brasília; 2010. [Série Cadernos da Indústria ABDI, 19].

10. acias-Chapula CA. O papel da informetria e da cienciometria e sua perspectiva nacional e internacional. CiInf. 1998;27(2):134-40.

11. Noronha DP, Maricato JM. Estudos métricos da informação: primeiras aproximações. EncontrosBibli. 2008;13(1):116-28.

12. Calero C, Buter R, Valdes CC, Noyons E. How to identify research groups using publication analysis: an example in the field of nanotechnology. Scientometrics. 2006;66(2):365-376.

13. Huang C, Notten A, Rasters N. Nanoscience and technology publications and patents: a review of social science studies and search strategies. J Technol Transfer. 2011;36(2):145-72.

14. Hullmann A, Meyer M. Publications and patents in nanotechnology. Scientometrics. 2003;58(3):507-27.

15. Kostoff, RN, StumpJA, Johnson D, Murday JS, Lau CGY, Tolles WM. The structure and infrastructure of the global nanotechnology literature. J Nanopart Res. 2006;8(3-4):301-21.

16. Kostoff R, Koytcheff R, Lau CGY. Global nanotechnology research metrics. Scientometrics. 2007;70(3):565-601.

17. Youtie J, Shapira P, Porter AL. Nanotechnology publications and citations by leading countries and blocs. J Nanopart Res. 2008;10(6):981-86.

18. Ostrowski AD, Martin T, Conti J, Hurt I, Harthorn BH. Nanotoxicology: characterizing the scientific literature, 2000-2007. J Nanopart Res. 2009;11(2):251-57.

19. Wang Q, Yang Z, Yang Y, Long C, Li H. A bibliometric analysis of research on the risk of engineering nanomaterials during 1999-2012. Sci Total Environ.2014;473:483-489. 
20. Guan J, Ma N. China's emerging presence in nanoscience and nanotechnology: acomparativebibliometric study of several nanoscience 'giants'. Res Policy. 2007;36(6):880-86.

21. lencar MSM, Porter A, Antunes AMS. Nanopatenting patterns in relation to product life cycle. Technol Forecast Soc. 2007;74(9):1661-80.

22. Rubi MP. Os princípios da política de indexação na análise de assunto para catalogação. In:Fujita MSL. (Org.). A indexação de livros: a percepção de catalogadores e usuários de bibliotecas universitárias. São Paulo: CulturaAcadêmica; 2009. p. 81-92.

23. Zitt M, BassecoulardE. Delineating complex scientific fields by an hybrid lexical-citation method: an application to nanosciences. Inform Process Manag. 2006;42(6)1513-31.

24. Mogoutov A,Kahane B. Data search strategy for science and technology emergence: a scalable and evolutionary query for nanotechnology tracking. Res Policy. 2007;36(6):893-903.

25. Porter AL, Youtie J, Shapira P, Schoeneck DJ. Refining search terms for nanotechnology. J Nanopart Res, 2008;10(5):715-28.

26. Ziman JM. Conhecimento público. Belo Horizonte: Itatiaia; São Paulo: USP; 1979.

27. BochnerR, AlencarMSM,Veiga VSO,Machado RR. A importância da padronização na informetria: um estudo exploratório na área de Saúde Pública. In: Anais do $13^{\circ}$ Encontro Nacional de Pesquisa em Ciência da Informação;2012 out. 28-31; Rio de Janeiro: Fiocruz; 2012. p. 1-16. 